Research Article

\title{
Preparation of Au-Ag Bimetals and Large-Size Porous Gold Nanostructured Materials
}

\author{
Xuewen Chen $\mathbb{D D}^{1,2}$ \\ ${ }^{1}$ Guangdong Engineering Polytechnic, Guangzhou 510520, China \\ ${ }^{2}$ Guangzhou Metals Association, Guangzhou 510520, China
}

Correspondence should be addressed to Xuewen Chen; lxd@hhtc.edu.cn

Received 15 July 2021; Accepted 18 November 2021; Published 13 December 2021

Academic Editor: Song Jiang

Copyright (c) 2021 Xuewen Chen. This is an open access article distributed under the Creative Commons Attribution License, which permits unrestricted use, distribution, and reproduction in any medium, provided the original work is properly cited.

Gold, silver, and other precious metals are very important nonferrous metals and have been widely applied in fields such as electronics, medicine, metallurgy, pharmaceuticals, and transportation. Adjustable properties of precious metals are mainly attributed to controlled synthesis of precious metals by structure, size, composition, and morphology. Synthesis of binary metals focuses on coordination of physical and chemical properties of metal elements in components, with the aim to give full play to the advantages of the two metals. Gold $(\mathrm{Au})$ and silver $(\mathrm{Ag})$ have similar lattice constants, which provide important theoretical basis for obtaining the binary bimetallic nanostructure of the two metals by coreduction at room temperature. Ag-Au alloy was prepared at different molar ratios of $\mathrm{Ag}^{+} / \mathrm{Au}^{\mathrm{III}}$, and the bimetallic nanomaterials obtained had similar $\mathrm{Ag} / \mathrm{Au}$ ratios to the molar ratio at reaction. This suggested that the bimetallic nanomaterials reacted completely, with the maximum average size in $\mathrm{Ag}_{90.1}-\mathrm{Au}_{9.9}$ and the minimum average size in $\mathrm{Ag}_{83.2}-\mathrm{Au}_{16.8}$ and $\mathrm{Ag}_{66.9}-\mathrm{Au}_{33.1}$. Due to the deficiency of conventional etching agents, the "regrowth etching" method was proposed in this study. Specifically, with $\mathrm{Au}^{\mathrm{I}}$ as the etching agent, the porous gold nanomaterials with the size of more than $300 \mathrm{~nm}$ were successfully prepared, achieving the regrowth etching effect and a good structural stability. According to the analysis based on the catalytic reduction reaction with p-nitrophenol, the properties of the large-size porous gold nanomaterials were related to the quantity and size of pores.

\section{Introduction}

1.1. Overview of Nanomaterial and Nanotechnology. Nanostructured material, "nanomaterial" for short, is a generic term for superfine granular materials with at least one dimension in the nanoscale. According to the definition adopted by the European Commission on October 18, 2011, "nanomaterial" means a natural, incidental, or manufactured material containing particles, in an unbound state or as an aggregate or as an agglomerate, where, for $50 \%$ or more of the particles in the number size distribution, one or more external dimensions is in the size range $1 \mathrm{~nm}-100 \mathrm{~nm}$. The European Commission suggests that basic particles of most nanomaterials are within this range, though materials beyond this range may also have the characteristics of nanomaterials. The definition aims to make the standard clear: a low threshold of nanoparticles will cover up the nanoproperties of the whole material, and $50 \%$ is an appropriate threshold. In addition, using the quantity ratio of nanoparticles instead of mass ratio as the measurement standard of nanomaterials can reflect the characteristics of nanomaterials better, because some nanomaterials have a very low density but still can show obvious characteristics of nanomaterials in the case of low mass ratio. Nanomaterials can be roughly divided into four categories: nanopowder, nanofiber, nanofilm, and nanoblock. Among them, the nanopowder features the longest development history and most mature technology and is the basis for the production of the other three.

Nanotechnology is a technology to study the properties and applications of materials with structural sizes ranging from $1 \mathrm{~nm}$ to $100 \mathrm{~nm}$. With the invention of scanning tunneling microscope in 1981, a new science with length $1 \mathrm{~nm}-100 \mathrm{~nm}$ as the research object was born, whose 
ultimate goal is to construct products with specific functions directly from atoms or molecules. Therefore, nanotechnology is actually a kind of science and technology that uses single atoms or molecules to make matters.

\subsection{Precious Metal Nanomaterials and Their Properties.} Precious metals generally refer to eight nonferrous metal elements, including gold, silver, and platinum group (including ruthenium, rhodium, palladium, osmium, iridium, and platinum). They are used in optoelectronics, energy, chemical, biomedical, and ceramic fields because of their unique optical, electrical, catalytic, and physicochemical properties. By adjusting the physical parameters and properties such as structure, morphology, size, and composition of precious metal nanocrystals, specific properties can be effectively obtained, realizing the application of precious metal nanomaterials. Precious metal nanomaterials refer to the new materials containing precious metals, whose size is less than $100 \mathrm{~nm}$ (or containing corresponding size of nanophase) obtained from precious metal products developed and produced by using nanotechnology.

Nanomaterials are powders, fibers, films, or blocks in the nanoscale. Scientific experiments have proved that when normal matter is processed to an extremely fine nanoscale, there will be specific surface effect, volume effect and quantum effect, and its optical, thermal, electrical, magnetic, mechanical, and even chemical properties will change significantly accordingly. Because of their small size, nanomaterials have very high surface energy and chemical activity and many special functions. Therefore, nanomaterials have superior properties that ordinary materials do not have.

Like nanomaterials, precious metal nanomaterials also have a series of special physical and chemical properties. The melting point of precious metal nanomaterials is obviously lower than that of conventional precious metal materials, which is due to the large specific surface area, high surface energy and interfacial energy, and less internal energy needed for melting of nanoparticles. The same is true of silver, gold, and platinum group metal nanoparticles. Precious metal nanoparticles have excellent catalytic activity and selectivity because of their larger specific surface area, higher surface energy, and surface crystal defects.

1.3. Preparation and Application of Precious Metal Nanomaterials. With the gradual weakening of the monetary function of precious metals such as gold, silver, platinum, and palladium, the industrial application of precious metals has increased rapidly. One of the reasons is that high and new technologies such as nanotechnology are constantly combined with traditional deep processing technology of precious metals, which has greatly expanded the application scope and quantity of precious metals in industry.

The preparation methods of precious metal nanomaterials can be divided into two categories: one is topdown, using macro- to submicroscopic methods, such as grinding, peeling, and etching; the other is bottom-up, using microscopic to submicroscopic methods, such as chemical synthesis, polymerization, and self-assembly. The former method mostly involves physical operations, while the latter involves chemical reactions. As there are many preparation methods of precious metal nanoparticles, and the preparation is easy, it has become one of the many advantages for precious metal nanoparticles to be widely studied and used.

At present, the research and application of nanotechnology mainly focus on materials and preparation, microelectronics and computer technology, medicine and health, aerospace and aviation, environment and energy, biotechnology, and agricultural products. Nanotechnology occupies a core position in the research and application of new materials. The equipment made of nanomaterials boasts of lighter weight, stronger hardness, longer life, lower maintenance cost, and more convenient design. Nanomaterials can also be used to produce materials with specific properties or materials that do not exist in nature, and to produce biomaterials and biomimetic materials.

Along with the constant development of modern science and technology, nanomaterials have played an increasingly important role in many fields. In particular, precious metal nanomaterials have attracted much attention because of their excellent physical and chemical properties [1]. The size and morphology of nanomaterials mainly depend on preparation methods. According to some study reports, synthesis of precious metallic nanocrystallines was controllable, and small-size monometallic nanomaterials had a high activity and excellent properties, but a poor stability, whereas large-size nanomaterials featured a stable structure, but their internal components were hard to interact [2-5]. Therefore, the regrowth etching technique was adopted in this study to etch $\mathrm{Au} / \mathrm{Ag}$ bimetallic nanomaterials, in the expectation of obtaining porous gold nanomaterials with a stable structure and large size. The specific study is as follows.

\section{Materials and Methods}

\subsection{Instruments and Reagents}

(1) The used instruments were as follows: ultrasonic apparatus, electronic scale, scanning electron microscope, beaker, stirrer, liquid ultraviolet spectrophotometer, centrifuge, transmission electron microscope, and cuvette

(2) The used reagents were as follows: silver nitrate, ammonium hydroxide, anhydrous ethanol, chloride acid (all were analytically pure), sodium borohydride, ascorbic acid, polyvinylpyrrolidone (PVP), p-nitrophenol, and deionized water

\section{Methods}

3.1. Preparation of Bimetallic Nanoparticles. After $4 \mathrm{~mL}$ of silver nitrate solution was mixed with $0.4 \mathrm{~g}$ of polyvinylpyrrolidone, the solution was dissolved in $5 \mathrm{~mL}$ of deionized water and stirred for $10 \mathrm{~min}$. After the solution was confirmed to be clear, $2 \mathrm{~mL}$ of ammonium hydroxide 
was added and stirred for $2 \mathrm{~min}$. Then, $2 \mathrm{~mL}$ of auric chloride solution was added to the mixture, and the total amount of $\mathrm{Ag}$ and $\mathrm{Au}$ must be $3 \mu \mathrm{mol}$. After $1 \mathrm{~mL}$ of vitamin $\mathrm{C}$ was added and reacted for $15 \mathrm{~min}$, the sample was cleaned with deionized water and then dispersed into $3 \mathrm{~mL}$ of deionized water for later use.

3.2. Synthesis of Au Nanoparticles. $4 \mathrm{~mL}$ of chloroauric acid solution and $0.4 \mathrm{~g}$ polyvinylpyrrolidone were added into $5 \mathrm{~mL}$ of deionized water and then fully stirred. After $1 \mathrm{~mL}$ vitamin $\mathrm{C}$ was added, the color of the solution changed accordingly. After reaction for $15 \mathrm{~min}$, the solution was cleaned with deionized water and then dispersed into deionized water for later use.

\subsection{Preparation of Porous Nano-Au}

(1) Appropriate amount of the vitamin C solution was added into the chloroauric acid solution. After the solution became transparent and colorless, the $\mathrm{Au}^{\mathrm{I}}$ solution was obtained. After the prepared $\mathrm{Ag}-\mathrm{Au}$ bimetallic nanoparticles were mixed with the $\mathrm{Au}^{\mathrm{I}}$ solution, the color would gradually change. Samples were collected at room temperature and cleaned and centrifuged with ammonium hydroxide and ionized water. The product obtained was dispersed into deionized water for later use.

(2) $\mathrm{By} \mathrm{Fe}^{3+}$ etching approach, the prepared $\mathrm{Ag}$-Au bimetallic nanoparticles were mixed with $20 \mathrm{mM}$ of ferric nitrate, and the color of the solution gradually faded. After reaction for half an hour, the samples were collected, cleaned with deionized water, and centrifuged. The product obtained was dispersed into deionized water for later use.

(3) By ammonium hydroxide erosion approach, the prepared Ag-Au bimetallic nanoparticles were mixed with appropriate amount of ammonium hydroxide. After reaction for $12 \mathrm{~h}$, the samples were collected, cleaned, and centrifuged. The final product was dispersed into deionized water for later use.

(4) By catalytic reduction with nitrophenol, $1 \mathrm{~mL}$ of p-nitrophenol and $1 \mathrm{~mL}$ of sodium borohydride were mixed. Then, the prepared Au-based nanoreactor was injected into the reaction system to obtain $3 \mathrm{~mL}$ of mixed solution. Finally, the mixed solution was placed in a quartz cuvette for an absorbance test, until the solution became colorless.

\section{Results}

4.1. Preparation of Bimetallic Precursor. Based on the closely matched lattice constants of $\mathrm{Ag}$ and $\mathrm{Au}$, nucleation and growth rates of $\mathrm{Ag}$ and $\mathrm{Au}$ were adjusted to reduce $\mathrm{Ag}-\mathrm{Au}$ alloy. The morphology of bimetallic nanomaterials obtained at different molar ratios $(10: 1,5: 1$, and $2: 1)$ of $\mathrm{Ag}^{+} / \mathrm{Au}^{\mathrm{III}}$ was observed by scanning electron microscope (SEM) as shown in Figure 1. In the chemical element components, the corresponding ratios of $\mathrm{Ag}$ to $\mathrm{Au}$ were 90.1:9.9, 83.2:16.8, and $66.9: 33.1$, respectively, which were close to the molar ratios at the reaction, indicating that the bimetallic nanomaterials obtained reacted completely.

After the average particle size of bimetallic nanomaterials above was determined, it was found that bimetallic nanomaterials synthesized at different molar ratios had certain differences. The average size of $\mathrm{Ag}_{90.1}-\mathrm{Au}_{9.9}, \mathrm{Ag}_{83.2}-\mathrm{Au}_{16.8}$, and $\mathrm{Ag}_{66.9}-\mathrm{Au}_{33.1}$ is as shown in Figure 2, with the maximum average size in $\mathrm{Ag}_{90.1}-\mathrm{Au}_{9.9}$ and the minimum average size in $\mathrm{Ag}_{83.2}-\mathrm{Au}_{16.8}$ and $\mathrm{Ag}_{66.9}-\mathrm{Au}_{33.1}$.

According to the analysis of SEM images and electron diffraction images of $\mathrm{Ag}_{66.9}-\mathrm{Au}_{33.1}$, the particles were polycrystalline, as shown in Figure 3(a). Based on dark-field transmission electron microscope images and bimetallic element distribution mapping images of $\mathrm{Ag}_{66.9}-\mathrm{Au}_{33.1}, \mathrm{Ag}$ and $\mathrm{Au}$ elements were widely distributed, as shown in Figures 3(b) and 3(c).

\section{Analysis of Etching Results}

5.1. Conventional Etching Agent. Conventionally, ammonium hydroxide, nitric acid, ferric nitrate, and other oxidizing agents were adopted for etching. For example, ammonium hydroxide and ferric nitrate were adopted for etching for $\mathrm{Ag}-\mathrm{Au}$ bimetal generally. However, in the process of operation, $\mathrm{Fe}^{3+}$ and ammonium hydroxide were difficult to etch $\mathrm{Ag}$ in $\mathrm{Ag}-\mathrm{Au}$ bimetal as expected. Through reaction between ferric nitrate and $\mathrm{Ag}_{66.9}-\mathrm{Au}_{33.1}$, the SEM image of the final product is shown in Figure 4(a). The analysis suggested that the whole structure of the product was collapsed and deformed, which was difficult to better maintain the porous gold structure. According to Figure 4(b) EDX image, the product had a significant Ag signal. The product featured a hollow structure, but with Ag uncleaned completely, which might be related to Au's involvement in $\mathrm{Fe}^{3+}$ reaction. With ammonium hydroxide as the etching agent, SEM and EDX images of the final product are shown in Figures 4(c) and 4(d). In the aerobic environment, ammonium hydroxide as a corrosive agent could react with $\mathrm{Ag}$ atoms to form $\left[\mathrm{Ag}\left(\mathrm{NH}^{3}\right) 2\right]^{+}$, making an etching effect. However, this study found that the product obtained after reaction with $\mathrm{Ag}_{66.9}-\mathrm{Au}_{33.1}$ did not achieve a good etching effect. The reason might be related to the slow reaction, and there was still as significant Ag signal.

5.2. Special Etching Agent: $A u^{I}$. It is difficult for the conventional etching agent to have a stable structure, and Au in residual components is not enough to maintain the largesize porous structure and results in obvious collapse and deformation. Therefore, a new-type etching agent that could obtain porous structures while providing complete etching is urgently needed, which is also an important way to obtain porous $\mathrm{Au}$. Based on this, $\mathrm{Au}^{\mathrm{III}}$ was first adopted as the etching agent in this study for the purpose of stabilizing the product structure. However, the following chemical reactions occurred simultaneously during the experiment: 


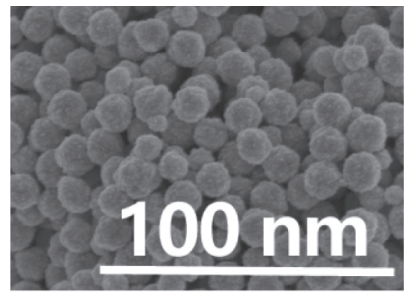

(a)

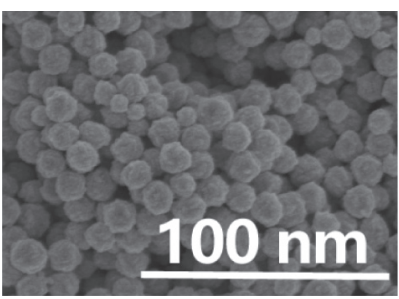

(b)

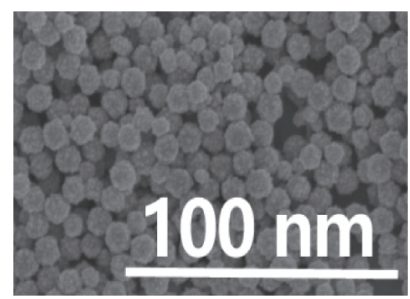

(c)

FIgURE 1: Low-power SEM images of product of $\mathrm{Ag}^{+} / \mathrm{Au}^{\mathrm{III}}$ at different molar ratios (a) $10: 1$; (b) $5: 1$; (c) $2: 1$.

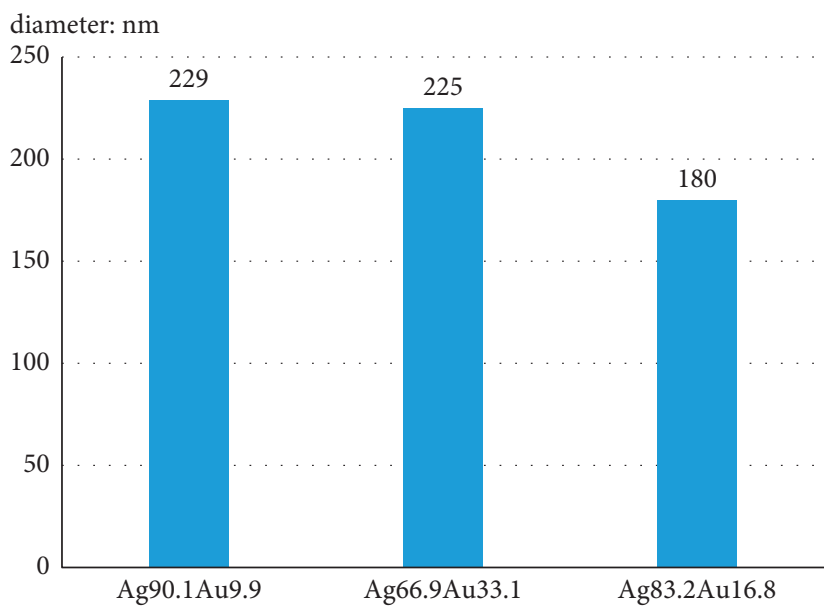

Figure 2: Average size of products of $\mathrm{Ag}^{+} / \mathrm{Au}^{\mathrm{III}}$ at different molar ratios.

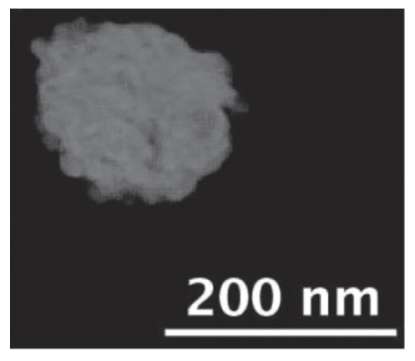

(a)

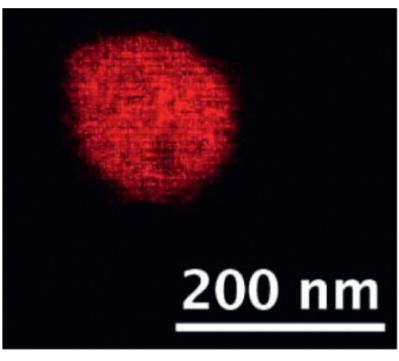

(b)

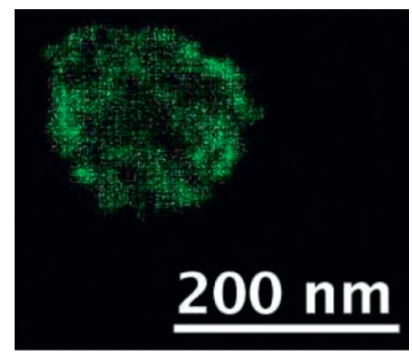

(c)

FIgURE 3: HADDF-STEM images and bimetallic element distraction mapping images of $\mathrm{Ag}_{66.9}-\mathrm{Au}_{33.1}$ : (a) HADDF-STEM image; (b) Ag; (c) Au.

$$
\begin{aligned}
3 A g+A u^{I I I} & =3 A g^{+}+A u, \\
2 A u+A u^{I I I} & =3 A u^{I} \\
A g+A u^{I} & =A u^{+}+A u .
\end{aligned}
$$

These reactions will inevitably lead to failure obtaining a good structure. Different oxidized states of $\mathrm{Au}$ have distinctive electrode potentials. Compared with $\mathrm{Au}^{\mathrm{III}}, \mathrm{Au}^{\mathrm{I}}$ has a lower valence state but a higher oxidizability and could avoid reactions (1) and (2). Therefore, $\mathrm{Au}^{\mathrm{I}}$ is more suitable than $\mathrm{Au}^{\mathrm{III}}$ as the etching agent for preparing porous gold nanostructures.
After $\mathrm{Ag}_{90.1}-\mathrm{Au}_{9.9}, \mathrm{Ag}_{83.2}-\mathrm{Au}_{16.8}$, and $\mathrm{Ag}_{66.9}-\mathrm{Au}_{33.1}$ were etched with $\mathrm{Au}^{\mathrm{I}}$, the three porous gold nanomaterials were identified as sample 1 , sample 2 , and sample 3 , respectively, as shown in SEM images in Figure 5. There were significant differences in morphology, quantity, and pore diameter of the porous materials with different bimetallic compositions.

After the average size of the previously mentioned three etched porous nanostructures was determined, it was found that the average particle sizes of sample $1\left(\mathrm{Ag}_{90.1}-\mathrm{Au}_{9.9}\right)$, sample 2 $\left(\mathrm{Ag}_{83.2}-\mathrm{Au}_{16.8}\right)$, and sample $3\left(\mathrm{Ag}_{66.9}-\mathrm{Au}_{33.1}\right)$ were $500 \mathrm{~nm}$, $400 \mathrm{~nm}$, and $300 \mathrm{~nm}$, respectively. This suggested that the materials obtained by $\mathrm{Au}^{\mathrm{I}}$ etching had a larger size, which accorded with the effect of regrowth etching, as shown in Figure 6. 


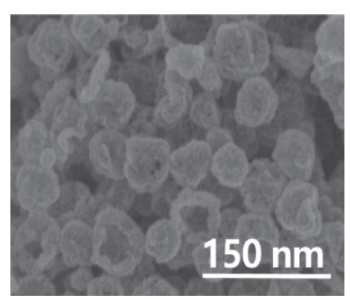

(a)

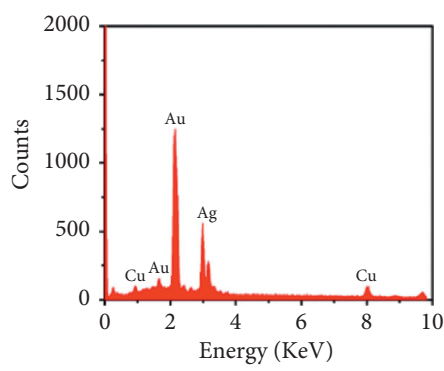

(b)

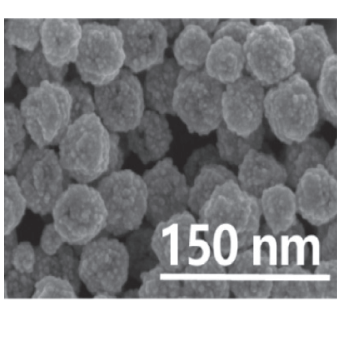

(c)

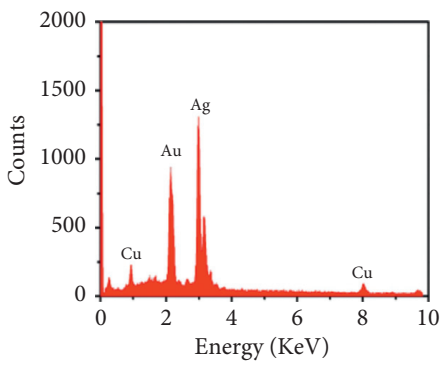

(d)

FIGURE 4: SEM and EDX images of product obtained with conventional etching agent: (a) SEM image; (b) EDX image; (c) SEM image; (d) EDX image.

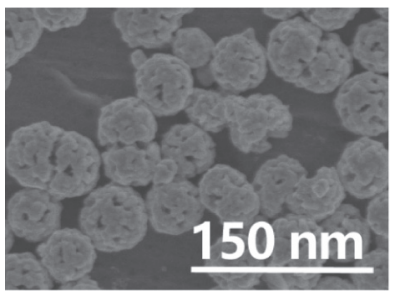

(a)

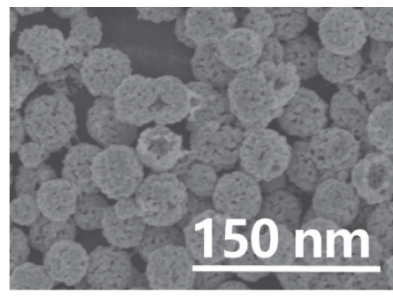

(b)

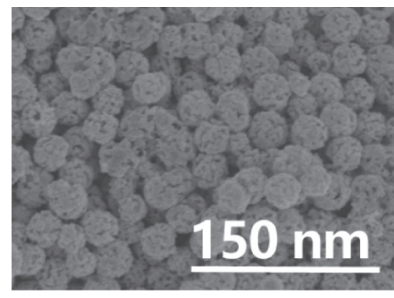

(c)

FIgURE 5: Porous nanostructures obtained by $\mathrm{Au}^{\mathrm{I}}$ etching: (a) $\mathrm{Ag}_{90.1}-\mathrm{Au}_{9.9}$; (b) $\mathrm{Ag}_{83.2}-\mathrm{Au}_{16.8}$; (c) $\mathrm{Ag}_{66.9}-\mathrm{Au}_{33.1}$.

TEM images of the products of the three samples after etching showed they all had the single-particle porosity. SAED images of $\mathrm{Ag}_{66.9}-\mathrm{Au}_{33.1}$ showed the particle was polycrystalline. Precursor $\mathrm{Ag}_{90.1}-\mathrm{Au}_{9.9}$ contained the highest property of Ag. Among the new samples obtained after etching, there were about 6 and $50 \mathrm{~nm}$ diameter pores in a single particle. Among precursor $\mathrm{Ag}_{83.2}-\mathrm{Au}_{16.8}$ samples, there were about 22 and $16 \mathrm{~nm}$ diameter pores in a single particle. Among $\mathrm{Ag}_{66.9}-\mathrm{Au}_{33.1}$ samples with less $\mathrm{Ag}$, there were about 35 and $11 \mathrm{~nm}$ diameter pores in a single particle. This indicated the different appearances of the final porous materials due to the difference in structural sites of Ag element in bimetallic components. Dark-field TEM images of porous materials obtained from $\mathrm{Ag}_{66.9}-\mathrm{Au}_{33.1}$ showed no $\mathrm{Ag}$ residue in porous gold nanomaterials, as shown in Figure 7.

The specific process of regrowth etching may be analyzed as follows. The replacement reaction between $\mathrm{Ag}$ atoms and $\mathrm{Au}^{\mathrm{I}}$ occurred on the $\mathrm{P}$ surface. With the disappearance of $\mathrm{Ag}$, Au grew on the bimetallic surface, and pores occurred on the surface. As the pores on the surface were diffused into the inner layer, their concentration increased, which led to the formation of a cavity. When $\mathrm{Ag}$ atoms and $\mathrm{Au}$ atoms in the inner layer were diffused to the surface layer, they reached a stable structure with the lowest energy, which improved the contact rate between $\mathrm{Ag}$ and $\mathrm{Au}^{\mathrm{I}}$ in the reaction liquid. Therefore, the original bimetallic structure disappeared, and the porous gold nanostructure was obtained finally.

\section{Catalytic Property of p-Nitrophenol}

Metal-based nanomaterials as a catalyst could promote p-nitrophenol reduction at the room temperature to a certain extent. Therefore, a typical catalytic reduction was

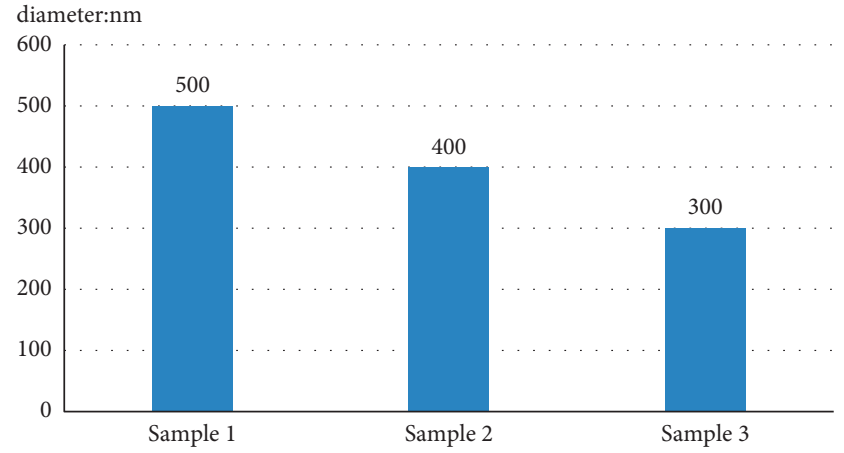

FIgURE 6: Average size of products obtained from three samples by $\mathrm{Au}^{\mathrm{I}}$ etching.

also adopted in this study to verify the properties of the Aubased nanomaterials obtained. In general, p-nitrophenol will not be reduced without a catalyst, even if a large amount of sodium borohydride is adopted. Nitrophenyl nitrate ions were formed in the solution, and their characteristic main peak was at about $400 \mathrm{~nm}$. However, after the effective catalyst was added, the main peak decreased, and the characteristic peak of p-nitrophenol was observed at about $292 \mathrm{~nm}$.

6.1. Catalysis with p-Nitrophenol. With sample 3 as the catalyst and porous nanoreactor as the reaction site, the UV absorbance spectrum after catalytic reduction with p-nitrophenol is shown in Figure 8(a). The whole process of p-nitrophenol reduction and aminophenol generation was complete in the porous reactor. During the $4 \mathrm{~min}$ of complete reaction, the main peak in the UV spectrum decreased 


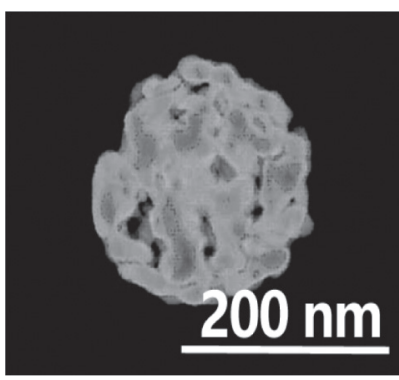

(a)

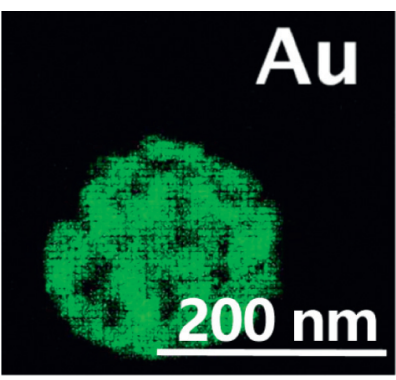

(b)

FIgURE 7: Dark-field TEM image of sample 3 and EDX mapping image of element Au: (a) TEM image; (b) EDX mapping image.

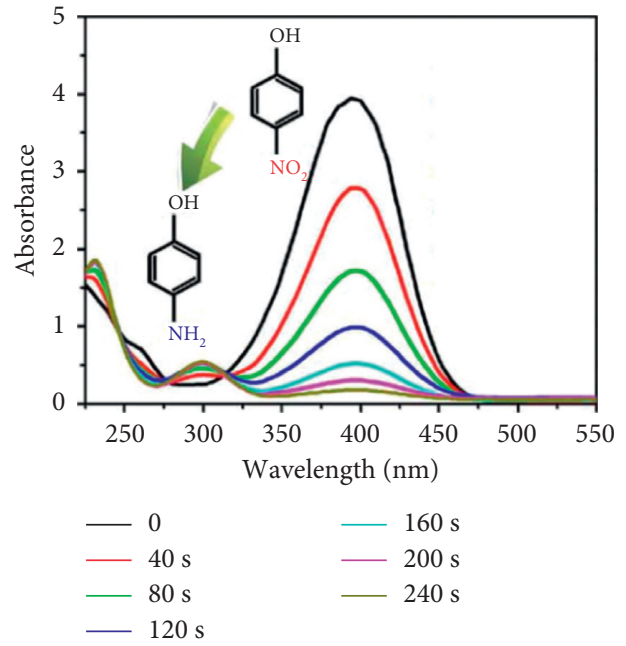

(a)

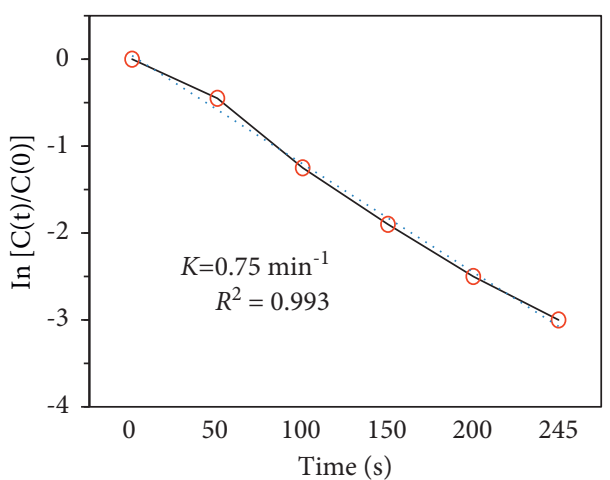

(b)

FIGURE 8: UV absorbance curves and catalytic time linear graph of sample 3 as catalyst: (a) UV absorbance curves; (b) relationship between $\ln [C(t) / C(0)]$ and time.

significantly, p-nitrophenols were all reduced, and the color of the solution became colorless and transparent. Figure 8(b) shows the kinetic process of sample 3 . The results showed that $\ln [C(t) / C(0)]$ was positively correlated with time, which accorded with the first-order reaction.

6.2. Comparison of Catalytic Results. In order to understand the catalytic property of porous gold nanomaterials prepared in this study, nitrophenol catalytic reduction tests were conducted for Au particles, three bimetals, and the first two corresponding products, respectively. The results showed that, with the increase of Au content in the components, the bimetallic materials showed a more significant catalytic effect, with a significantly shorter time required. Among the porous samples, the large-pore-diameter sample 1 had a worse catalytic effect, whereas small-pore-diameter samples 2 and 3 had a better catalytic effect, as shown in Figure 9.

The catalytic time of Au was more than $2 \mathrm{~h}$, while the catalytic times required by $\mathrm{Ag}_{90.1}-\mathrm{Au}_{9.9} ; \mathrm{Ag}_{83.2}-\mathrm{Au}_{16.8}$; $\mathrm{Ag}_{66.9}-\mathrm{Au}_{33.1}$ were $31 \mathrm{~min}, 18 \mathrm{~min}$, and $16 \mathrm{~min}$, respectively. Therefore, the time required by porous materials was significantly shorter. Specifically, the times required by samples 1 and 2 were $10 \mathrm{~min}$ and $6 \mathrm{~min}$, respectively. This suggested that the properties of porous gold nanomaterials were related to the quantity and size of pores.

\section{Discussion}

Energy, materials, and information are important pillars of modern science and technology, among which material technology is the key to solve energy and environment problems [6]. Nanotechnology plays a very important role in such fields as medicine, optics, and catalysis. Gold and silver are the most common precious metals. New precious metal materials prepared by nanotechnology have special physical and chemical properties and become an important part of nanotechnology studies [7]. Some studies reported that hollow, porous, or framed precious metal nanomaterials differed greatly from solid nanoparticles in terms of structure, with a larger effective contact area, lower overall density, and better liquid fluidity [8-10]. Therefore, in the actual synthesis process, these nanomaterials as the catalytic reaction reactor can effectively increase the contact area and involve gas and liquid more easily, so as to promote the reaction [11]. Due to their excellent properties, porous materials are widely used in various fields. In 


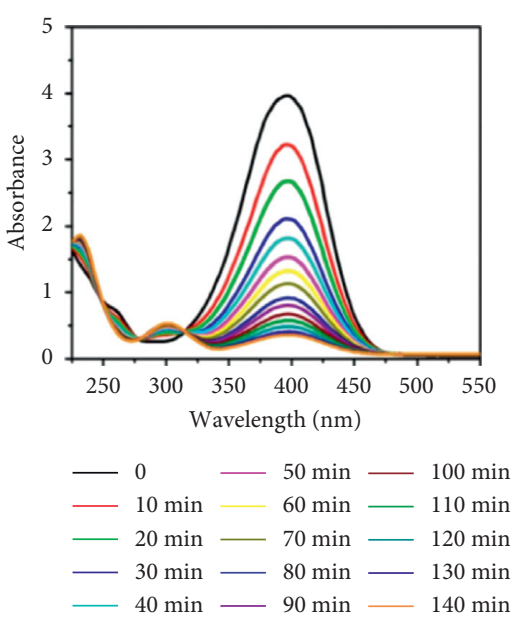

(a)

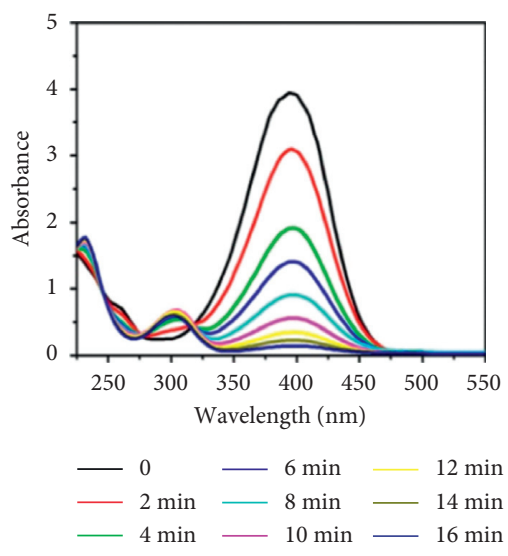

(d)

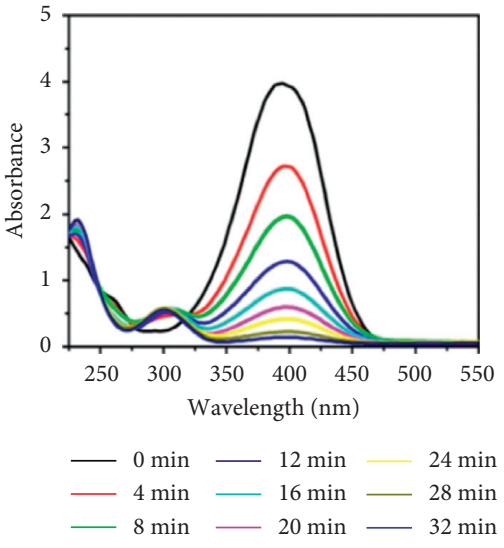

(b)

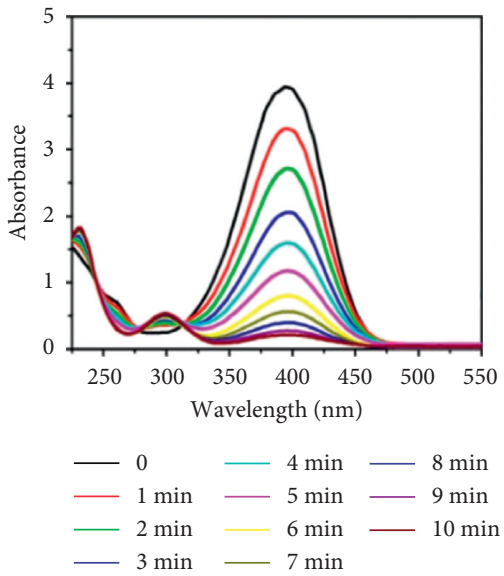

(e)

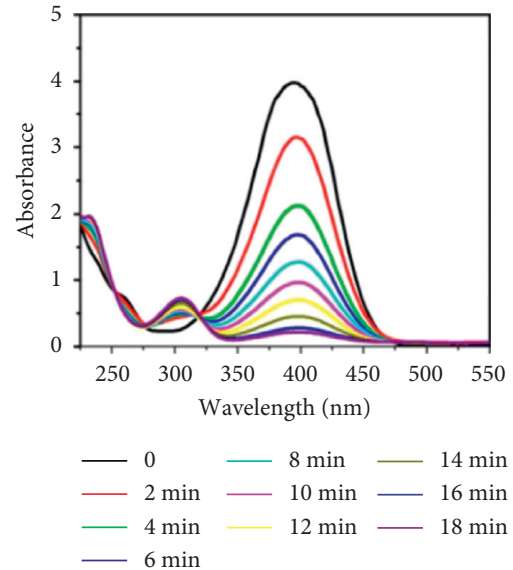

(c)

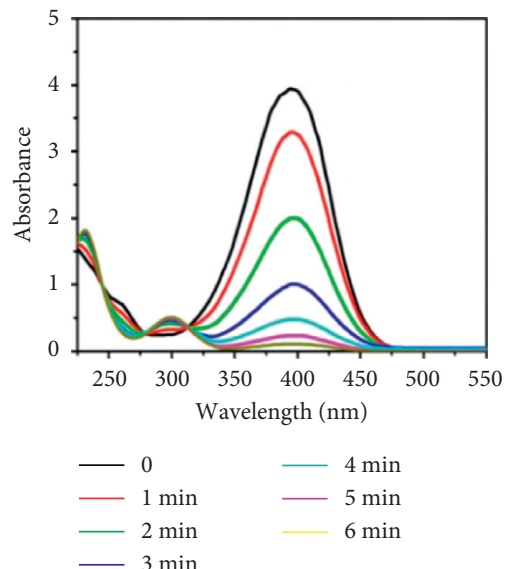

(f)

FigURE 9: UV absorbance spectrum of catalytic reduction with different Au-based catalysts: (a) $\mathrm{Au}$ particle; (b) $\mathrm{Ag}_{90.1}-\mathrm{Au}_{9.9}$; (c) $\mathrm{Ag}_{83.2}-\mathrm{Au}_{16.8} ;$ (d) $\mathrm{Ag}_{66.9}-\mathrm{Au}_{33.1}$; (e) sample 1; (f) sample 2.

appropriate solutions, metal salt can be reduced to obtain monometal nanostructures. Accordingly, two different metals can be reduced to get bimetallic nanostructures. However, because of the differences between the two metals in chemical properties and reduction potential, it is difficult to control their reduction and nucleation processes simultaneously [12]. Coreduction synthesis, replacement synthesis, and thermal decomposition synthesis are the most commonly used methods for preparing bimetallic nanomaterials.

Etching bimetallic materials by replacement reaction is a widely used and effective approach to obtain monometallic porous nanostructures at present. During the whole process of operation, metal A with the reduction property is adopted as the template, and metal $B$ ion with the oxidization property is adopted as the etching agent in general. While B grows towards the outer layer, $A$ atom in the inner layer is also diffused outward. Finally, B deposits on the surface of A and forms a shell [13]. In this way, the alloy of hollow spheres is obtained. During this process, the etching agent not only adjusts the inner cavity but also modifies the outer shell [14]. According to the application advantages of replacement reaction, during the specific process of operation, bimetallic materials can be etched to prepare large-size monometallic nanomaterials, such as Au nanotubes, Au nanocages, and $\mathrm{Au} / \mathrm{Pt}$. These etched precursors all have a frame-like structure [15]. In brief, hollow $\mathrm{Ag} / \mathrm{Au}$ nanoshells are transformed into porous nanocages. In practice, it is difficult to prepare large-size spherical porous nanostructures (with a diameter of more than $100 \mathrm{~nm}$ ). For instance, monometallic $\mathrm{M}$ template is generally adopted for Au nanomaterials, and the Au-M nanoshell structure is formed after displacement reaction. Then, residual metal $\mathrm{A}$ is cleaned by etching. However, it is difficult to obtain large-size spherical porous materials by this operation [16]. The challenges mainly exist in the following aspects. First, although the size increases after replacement reaction, it cannot be further increased. Second, the increase in the size of the selected etching templates causes the decrease in the composition etched materials and structural stability and the possibility of collapse. Third, the porous gold structures obtained by bimetallic etching are prone to have silver residues, and the reactants may cause blocking when passing through pores [17]. 
Based on this, the "regrowth etching" method was proposed in this study, with the aim to reduce collapse caused by structural instability. First, Ag-Au bimetallic materials were prepared through adjustment of the nucleation and growth rates of $\mathrm{Ag}$ and $\mathrm{Au}$. Although all of the prepared $\mathrm{Ag}_{90.1}-\mathrm{Au}_{9.9}$, $\mathrm{Ag}_{83.2}-\mathrm{Au}_{16.8}$, and $\mathrm{Ag}_{66.9}-\mathrm{Au}_{33.1}$ bimetallic nanomaterials reacted completely, $\mathrm{Ag}$ was high in the precursor and occupied more sites. Then, $\mathrm{Au}^{\mathrm{I}}$ was adopted as the etching agent to further trigger the replacement reaction with Ag, which not only obtained large-size porous gold nanomaterials with a better stability but also effectively avoided collapse and deformation. The catalytic reduction reaction with p-nitrophenol confirmed that hollow porous materials have a more advantageous catalytic activity. The enhanced diffusion effect of porous structure is mainly manifested as follows. First, the large-volume-fraction interface in the structure provides an important way for short-cycle diffusion. Second, the particle interfaces and cavities after the replacement reaction have high-density defects. Third, due to the driving force, the stress and deformation in the crystals enhance the diffusion effect. Fourth, pores in materials lead to capillary phenomenon, which is more significant in materials with small-diameter pores in general. Because porous nanomaterials are a reaction site, the fluid transfer process is mainly completed through the pores. Small-size pores are easy to be blocked by reactants or products, while a large number of pores can provide multiple channels to transfer these substances, so as to effectively reduce pore blocking. At present, it is generally believed that nanomaterials with a large number of $10 \mathrm{~nm}$-diameter pores have a good catalytic effect and are excellent catalytic materials [18].

In conclusion, the regrowth etching method was adopted in this study to successfully prepare large-size porous gold nanomaterials with a good structural stability. The properties of porous gold nanomaterials were improved through adjustment of the size and quantity of pores to a certain degree. Applied as a catalytic reaction site, these materials have more outstanding properties than solid particles and are expected to play important roles in disease detection, catalysis, and biomedicine.

\section{Conclusion}

$\mathrm{Ag}-\mathrm{Au}$ alloy was prepared at different molar ratios of $\mathrm{Ag}^{+} /$ $\mathrm{Au}$, and the bimetallic nanomaterials obtained had similar $\mathrm{Ag} / \mathrm{Au}$ ratios to the molar ratio at reaction. This suggested that the bimetallic nanomaterials reacted completely, with the maximum average size in $\mathrm{Ag}_{90.1}-\mathrm{Au}_{9.9}$ and the minimum average size in $\mathrm{Ag}_{83.2}-\mathrm{Au}_{16.8}$ and $\mathrm{Ag}_{66.9}-\mathrm{Au}_{33.1}$.

Due to the deficiency of conventional etching agents, the "regrowth etching" method was proposed in this study. Specifically, with AuI as the etching agent, the porous gold nanomaterials with the size of more than $300 \mathrm{~nm}$ were successfully prepared, achieving the regrowth etching effect and a good structural stability.

According to the analysis based on the catalytic reduction reaction with p-nitrophenol, the properties of the large-size porous gold nanomaterials were related to the quantity and size of pores.

\section{Data Availability}

The data used to support the findings of this study are included within the article.

\section{Conflicts of Interest}

The authors declare that they have no conflicts of interest to declare.

\section{References}

[1] Yu. Guan, Study of Preparation and Catalysis of Titanium Dioxide Nanoribbon-Supported Bimetallic Catalyst and its Photocatalytic Property, Shandong University, Shandong, 2013.

[2] F. Shaojie, D. Zhao, F. Xie, W. Xiufang, and Z. Jun, Study of Synthesis and Structural Adjustment of Inorganic Nanomaterials and Their Functions, Anhui Jianzhu University, Hefei, China, 2017.

[3] Qi Zhou, Z. Li, and B. Zheng, "Preparation of nanoporous bimetallic oxide $\mathrm{NiCo} 2 \mathrm{O} 4$ and its electrochemical property," Chinese Journal of Inorganic Chemistry, vol. 34, no. 6, pp. 1103-1109, 2018.

[4] Y. Li, M. Zhang, X. Wang, and W. Li, "Advance in studies of preparation method of nanoporous metals and their mechanical properties," Journal of Aeronautical Materials, vol. 38, no. 05, pp. 10-23, 2018.

[5] G. Tao, Z. Cheng, D. Zhang, and F. Hang, "Preparation of COdoped zinc oxide porous materials derived from bimetallic MOF and their gas-sensitive properties," Journal of Functional Materials, vol. 51, no. 9, pp. 9185-9192, 2020.

[6] X. Hu, Y. Yin, W. Liu et al., "Preparation of cobalt phosphide @ iron-zinc bimetallic phosphide multistage nano-composites and their electrocatalytic water decomposition properties," Chinese Journal of Catalysis, vol. 40, no. 7, pp. 1093-1104, 2019.

[7] M. Mceachran, D. Keogh, B. Pietrobon et al., "Ultrathin gold nanoframes through surfactant-free templating of faceted pentagonal silver nanoparticles," Journal of the American Chemical Society, vol. 133, no. 21, pp. 8066-8069, 2011.

[8] J. Li, Construction of Transition Metal-Based Three-Dimensional Nanostructures and Their Electrochemical Properties, Qingdao University of Science and Technology, Shandong, 2019.

[9] T. Zhang, Preparation of Porous Precious Metal Alloy Nanomaterials and Their Optical/Electrocatalytic Properties, Anhui: University of Science and Technology of China, Huainan, China, 2019.

[10] C. Tian, Study of Synthesis of Nanoporous Metal/Graphene Composites and Their Electrochemical Properties, China University of Mining and Technology, Beijing, China, 2019.

[11] W. Shaozhen, K. Wang, H. Long et al., "A highly efficient, clean-surface, porous platinum electrocatalyst and the inhibition effect of surfactants on catalytic activity," Chemistry - A European Journal, vol. 19, no. 1, p. 416, 2013.

[12] Qi Zhou, B. Zheng, Z. Li, W. Yafei, and F. Jiwei, "Dealloying preparation of nanoporous bimetallic oxide NiMoO4 and its electrochemical properties," Chinese Journal of Inorganic Chemistry, vol. 33, no. 8, pp. 1416-1422, 2017.

[13] H. Luo, C. Xu, H. Wu, and Z. Yun, "Preparation of silicon/ carbon composites derived from organic frameworks of cobalt-iron bimetals and their electrochemical properties," Sichuan Nonferrous Metals, no. 2, pp. 48-51, 2020. 
[14] S. Zheng, Z. Ma, H. Yuan, L. Jinping, and W. Xiaoguang, "Study of preparation of self-supported nanoporous Al-doped $\mathrm{NiCu}-\mathrm{S}$ electrode and its electrolyzed water hydrogen evolution," Journal of Taiyuan University of Technology, vol. 52, no. 1, pp. 29-37, 2021.

[15] X. Tan, A. Tahini Hassan, and C. Smith Sean, "Conductive boron-doped graphene as an ideal material for electrocatalytically switchable and high-capacity hydrogen storage," ACS applied materials \& interfaces, vol. 48, 2016.

[16] C. Sundaram, L. Yao, L. Deng et al., "Recent advances in metal sulfides: from controlled fabrication to electrocatalytic, photocatalytic and photoelectrochemical water splitting and beyond," Chemical Society Reviews, vol. 48, no. 15, pp. 4178-4280, 2019.

[17] Z. Yan, D. Wu, and Z. Wang, "Advance in studies of preparation of nanoscale zero-valent iron and its application," Applied Chemical Industry, vol. 68, no. 03, Article ID 012052, 2021.

[18] Y. X. Bao, B. W. Hao, X. Wang, and X. W. Lou, "One-pot synthesis of cubic PtCu3 nanocages with enhanced electrocatalytic activity for the methanol oxidation reaction," Journal of the American Chemical Society, vol. 134, no. 34, pp. 13934-13937, 2012. 